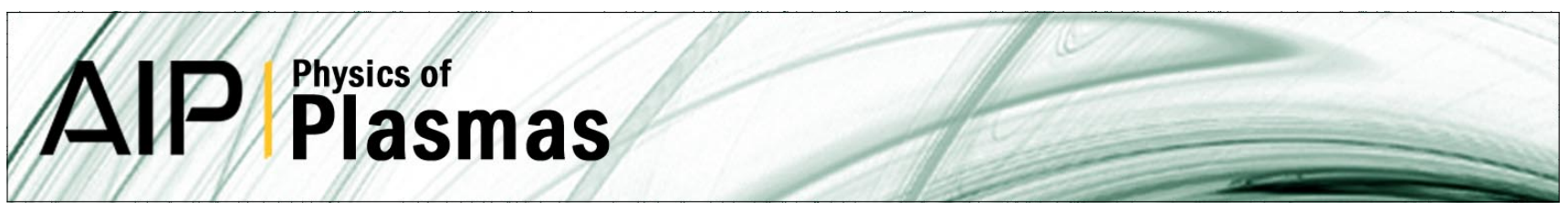

\title{
Electromagnetic weak turbulence theory revisited
}

P. H. Yoon, L. F. Ziebell, R. Gaelzer, and J. Pavan

Citation: Phys. Plasmas 19, 102303 (2012); doi: 10.1063/1.4757224

View online: http://dx.doi.org/10.1063/1.4757224

View Table of Contents: http://pop.aip.org/resource/1/PHPAEN/v19/i10

Published by the American Institute of Physics.

\section{Related Articles}

Freely decaying turbulence in two-dimensional electrostatic gyrokinetics

Phys. Plasmas 19, 122305 (2012)

Magnetic turbulence suppression by a helical mode in a cylindrical geometry

Phys. Plasmas 19, 122304 (2012)

Effect of secondary convective cells on turbulence intensity profiles, flow generation, and transport

Phys. Plasmas 19, 112506 (2012)

Asymmetric chiral alignment in magnetized plasma turbulence

Phys. Plasmas 19, 112301 (2012)

Three-dimensional modeling of beam emission spectroscopy measurements in fusion plasmas

Rev. Sci. Instrum. 83, 113501 (2012)

\section{Additional information on Phys. Plasmas}

Journal Homepage: http://pop.aip.org/

Journal Information: http://pop.aip.org/about/about_the_journal

Top downloads: http://pop.aip.org/features/most_downloaded

Information for Authors: http://pop.aip.org/authors

\section{ADVERTISEMENT}






\title{
Electromagnetic weak turbulence theory revisited
}

\author{
P. H. Yoon, ${ }^{1, a)}$ L. F. Ziebell, ${ }^{2, b)}$ R. Gaelzer, ${ }^{3, c)}$ and J. Pavan ${ }^{3, d)}$ \\ ${ }^{1}$ IPST, University of Maryland, College Park, Maryland 20742, USA \\ ${ }^{2}$ Instituto de Física, UFRGS, Porto Alegre, RS, Brazil \\ ${ }^{3}$ Instituto de Física e Matemática, UFPel, Pelotas, RS, Brazil
}

(Received 5 September 2012; accepted 18 September 2012; published online 9 October 2012)

The statistical mechanical reformulation of weak turbulence theory for unmagnetized plasmas including fully electromagnetic effects was carried out by Yoon [Phys. Plasmas 13, 022302 (2006)]. However, the wave kinetic equation for the transverse wave ignores the nonlinear threewave interaction that involves two transverse waves and a Langmuir wave, the incoherent analogue of the so-called Raman scattering process, which may account for the third and higher-harmonic plasma emissions. The present paper extends the previous formalism by including such a term. (C) 2012 American Institute of Physics. [http://dx.doi.org/10.1063/1.4757224]

\section{INTRODUCTION}

The formulation of weak turbulence theory began in the late 1950 s to early 1970 s, largely by scientists from the former Soviet Union. Most of the discussions found in the literature, however, are concerned with electrostatic problems in unmagnetized plasmas. ${ }^{1-16}$ The generalization to include transverse electromagnetic waves is of obvious importance in view of its applicability to the solar type II and III radio bursts. In the literature, electromagnetic effects on the weak turbulence theory are often incorporated only in a formal sense. ${ }^{8-10,12,17-21}$ In many cases, the fundamental equations are given only in terms of formal nonlinear coupling coefficients defined in terms of susceptibility tensors, but unless these susceptibilities are explicitly computed under appropriate approximations, the formalism remains impractical.

One of the present authors (P.H.Y.) revisited the problem of electromagnetic weak turbulence theory, and systematically re-derived the set of equations that may readily be solved by numerical means. ${ }^{22}$ The basic set of equations describe the dynamical evolution of the electron and ion velocity (or momentum) distribution functions, time evolution and interactions of Langmuir, ion-acoustic, and transverse electromagnetic waves, as well as various linear and nonlinear interactions among waves and particles.

Note that Ref. 22 can readily be compared against the formalism based upon the semi-classical approach, pioneered in large part by Tsytovich. ${ }^{23}$ The semi-classical method is a heuristic approach that bypasses the rigorous but tedious perturbation analysis by relying on intuitive reasoning, and the method has been extensively employed in the study of plasma emission by Melrose and the University of Sydney group. ${ }^{23-39}$ Reference 22 establishes the equivalence of the statistical mechanical approach and the semi-classical formalism.


yoonp@umd.edu.

b) Electronic address: ziebell@if.ufrgs.br.

${ }^{\text {c)} E l e c t r o n i c ~ a d d r e s s: ~ r u d i @ u f p e l . e d u . b r . ~}$

${ }^{\mathrm{d})}$ Electronic address: joel.pavan@ufpel.edu.br.
}

It turns out, however, that the transverse wave kinetic equation in Ref. 22 ignores the nonlinear three-wave interaction that involves the merging and decay of two transverse waves and a Langmuir wave, which corresponds to the incoherent counterpart of the so-called Raman scattering process. Such an interaction may account for the third and higherharmonic plasma emissions. Also, it turns out that the transverse wave kinetic equation has a sign mistake in one of the terms that describe the spontaneous scattering effects. The purpose of the present paper is to extend the formalism to include higher-harmonic emissions as well as to correct the sign mistake.

\section{THEORETICAL DISCOURSE}

A convenient starting point is the set of formal equations derived in Ref. 22. These are formal particle kinetic equation and formal wave kinetic equations expressed in terms of linear and nonlinear susceptibility tensors. First, the formal particle kinetic equation can be found in Eq. (44) of Ref. 22, where the kinetic equation for particle species $a$ contains both the collisional (i.e., Balescu-Lenard) term and FokkerPlanck operator determined by electrostatic plasma eigenmodes, Langmuir $(L)$ and ion-sound $(S)$ waves

$$
\begin{aligned}
\frac{\partial f_{a}(\mathbf{v})}{\partial t}= & \sum_{b} \frac{2 \hat{n}^{2} e_{a}^{2} e_{b}^{2}}{m_{a}^{2}} \int d \mathbf{k} \int d \mathbf{v}^{\prime} \mathbf{k} \cdot \frac{\partial}{\partial \mathbf{v}} \mathcal{P} \frac{\delta\left(\mathbf{k} \cdot \mathbf{v}-\mathbf{k} \cdot \mathbf{v}^{\prime}\right)}{k^{4}\left|\epsilon_{\|}(\mathbf{k}, \mathbf{k} \cdot \mathbf{v})\right|^{2}} \\
& \times\left(f_{b}\left(\mathbf{v}^{\prime}\right) \mathbf{k} \cdot \frac{\partial f_{a}(\mathbf{v})}{\partial \mathbf{v}}-\frac{m_{a}}{m_{b}} f_{a}(\mathbf{v}) \mathbf{k} \cdot \frac{\partial f_{b}\left(\mathbf{v}^{\prime}\right)}{\partial \mathbf{v}^{\prime}}\right) \\
& +\frac{\pi e_{a}^{2}}{m_{a}^{2}} \sum_{\sigma= \pm 1} \sum_{\alpha=L, S} \int \frac{d \mathbf{k}}{k^{2}} \mathbf{k} \cdot \frac{\partial}{\partial \mathbf{v}} \delta\left(\sigma \omega_{\mathbf{k}}^{\alpha}-\mathbf{k} \cdot \mathbf{v}\right) \\
& \times\left(\frac{m_{a} \sigma \omega_{\mathbf{k}}^{\alpha}}{4 \pi^{2}} f_{a}(\mathbf{v})+I_{\mathbf{k}}^{\sigma \alpha} \mathbf{k} \cdot \frac{\partial f_{a}(\mathbf{v})}{\partial \mathbf{v}}\right) .
\end{aligned}
$$

Here, $f_{a}(\mathbf{v})$ is the one-particle distribution function for species labeled $a$ (where $a=e$ for the electrons and $a=i$ for the ions, or the protons). The distribution function is normalized as $\int d \mathbf{v} f_{a}(\mathbf{v})=\hat{n}$, where $\hat{n}$ represents the particle 
number density (which is the same for both the electrons and ions by virtue of the quasi-neutrality). Other notations are standard, $e_{a}$ being the electric charge $\left(e_{a}=-e\right.$ for the electrons and $e_{a}=e$ for the protons), and $m_{a}$ representing the mass for species $a$.

Note that the Balescu-Lenard collisional integral (the first term on the right-hand side) should become important when the particles are close to thermal equilibrium. In contrast, the Fokker-Planck term dictated by the wave-particle interaction condition, $\delta\left(\sigma \omega_{\mathbf{k}}^{\alpha}-\mathbf{k} \cdot \mathbf{v}\right)$, is supposed to be important when enhanced plasma eigenmodes are present, such as in the presence of a free energy source that drives the instability. In the collisional term the linear dielectric constant $\epsilon_{\|}(\mathbf{k}, \omega)$ appears in the denominator. This quantity shall be defined later together with nonlinear susceptibilities.

In the Fokker-Planck term, the longitudinal electric field intensities, $I_{\mathbf{k}}^{\sigma \alpha}$ for $\alpha=L, S$, are defined by $\left\langle\delta E_{\|}^{2}\right\rangle_{\mathbf{k}, \omega}$ $=\sum_{\sigma= \pm 1} \sum_{\alpha=L, S} I_{\mathbf{k}}^{\sigma \alpha} \delta\left(\omega-\sigma \omega_{\mathbf{k}}^{\alpha}\right)$. The transverse mode does not affect the particles since the fast waves cannot resonate with the particles. However, for later purposes, we may express the spectral electric field intensity associated with the fast transverse wave as $\left\langle\delta E_{\perp}^{2}\right\rangle_{\mathbf{k} \omega}=\sum_{\sigma= \pm 1} I_{\mathbf{k}}^{\sigma T}$ $\delta\left(\omega-\sigma \omega_{\mathbf{k}}^{T}\right)$. In full tensor notation, the two-body cumulant of the total electric field fluctuations is given by

$$
\left\langle\delta E_{i} \delta E_{j}\right\rangle_{\mathbf{k} \omega}=\frac{k_{i} k_{j}}{k^{2}}\left\langle\delta E_{\|}^{2}\right\rangle_{\mathbf{k} \omega}+\frac{1}{2}\left(\delta_{i j}-\frac{k_{i} k_{j}}{k^{2}}\right)\left\langle\delta E_{\perp}^{2}\right\rangle_{\mathbf{k} \omega} .
$$

Of course, the magnetic field fluctuation is related to the transverse electric field fluctuation by $\delta \mathbf{B}_{\mathbf{k}, \omega}=c \mathbf{k} \times \delta \mathbf{E}_{\mathbf{k}, \omega} / \omega$. The linear dispersion relations for electrostatic (Langmuir $L$, and ion-sound $S$ ) and transverse electromagnetic $(T)$ modes are given by

$$
\begin{aligned}
& \omega_{\mathbf{k}}^{L}=\omega_{p e}\left(1+3 k^{2} \lambda_{D}^{2} / 2\right)^{-1 / 2}, \\
& \omega_{\mathbf{k}}^{S}=k c_{S}\left(1+3 T_{e} / T_{i}\right)^{1 / 2}\left(1+k^{2} \lambda_{D}^{2}\right)^{-1 / 2}, \\
& \omega_{\mathbf{k}}^{T}=\left(\omega_{p e}^{2}+c^{2} k^{2}\right)^{1 / 2},
\end{aligned}
$$

where $\omega_{p e}=\left(4 \pi \hat{n} e^{2} / m_{e}\right)^{1 / 2}$ is the plasma frequency, $\lambda_{D}=$ $\left[T_{e} /\left(4 \pi \hat{n} e^{2}\right)\right]^{1 / 2}$ is the Debye length, $T_{e}$ and $T_{i}$ are electron and ion temperatures, respectively, and $c_{S}=\left(T_{e} / m_{i}\right)^{1 / 2}$ represents the ion-sound speed.

The next set of equations is formal wave kinetic equations for electrostatic (or longitudinal) modes $\alpha=L, S$, and the transverse wave kinetic equation. Reference 22 already derived these equations, but since the purpose of the present paper is correct one of the sign mistakes as well as to include certain nonlinear interaction terms that were missing in the original paper, we shall begin by reproducing a formal set of wave kinetic equations expressed in terms of exact linear and nonlinear susceptibility tensors. The relevant equations can be found in Eqs. (31) and (32) of Ref. 22. First, the formal equation for longitudinal modes is given by Eq. (31) of Ref. 22

$$
\begin{aligned}
& \frac{\partial I_{\mathbf{k}}^{\sigma \alpha}}{\partial t}=-\frac{2 \operatorname{Im} \epsilon_{\|}\left(\mathbf{k}, \sigma \omega_{\mathbf{k}}^{\alpha}\right)}{\epsilon_{\|}^{\prime}\left(\mathbf{k}, \sigma \omega_{\mathbf{k}}^{\alpha}\right)} I_{\mathbf{k}}^{\sigma \alpha}+\sum_{a} \frac{4 e_{a}^{2}}{k^{2}\left|\epsilon_{\|}^{\prime}\left(\mathbf{k}, \sigma \omega_{\mathbf{k}}^{\alpha}\right)\right|^{2}} \int d \mathbf{v} \delta\left(\sigma \omega_{\mathbf{k}}^{\alpha}-\mathbf{k} \cdot \mathbf{v}\right) f_{a}(\mathbf{v})-\frac{4 \pi}{\epsilon_{\|}^{\prime}\left(\mathbf{k}, \sigma \omega_{\mathbf{k}}^{\alpha}\right)} \sum_{\sigma^{\prime}, \sigma^{\prime \prime}} \sum_{\beta, \gamma} \int d \mathbf{k}^{\prime} \mid \frac{k_{i} k_{j}^{\prime}\left(\mathbf{k}-\mathbf{k}^{\prime}\right)_{k}}{k k^{\prime}\left|\mathbf{k}-\mathbf{k}^{\prime}\right|} \\
& \times\left.\chi_{i j k}^{(2)}\left(\mathbf{k}^{\prime}, \sigma^{\prime} \omega_{\mathbf{k}^{\prime}}^{\beta} \mid \mathbf{k}-\mathbf{k}^{\prime}, \sigma^{\prime \prime} \omega_{\mathbf{k}-\mathbf{k}^{\prime}}^{\gamma}\right)\right|^{2}\left(\frac{I_{\mathbf{k}-\mathbf{k}^{\prime}}^{\sigma^{\prime \prime} \gamma} I_{\mathbf{k}}^{\sigma \alpha}}{\epsilon_{\|}^{\prime}\left(\mathbf{k}^{\prime}, \sigma^{\prime} \omega_{\mathbf{k}^{\prime}}^{\beta}\right)}+\frac{I_{\mathbf{k}^{\prime}}^{\sigma^{\prime} \beta} I_{\mathbf{k}}^{\sigma \alpha}}{\epsilon_{\|}^{\prime}\left(\mathbf{k}-\mathbf{k}^{\prime}, \sigma^{\prime \prime} \omega_{\mathbf{k}-\mathbf{k}^{\prime}}^{\gamma}\right)}-\frac{I_{\mathbf{k}^{\prime}}^{\sigma^{\prime} \beta} I_{\mathbf{k}-\mathbf{k}^{\prime}}^{\sigma^{\prime \prime} \gamma}}{\epsilon_{\|}^{\prime}\left(\mathbf{k}, \sigma \omega_{\mathbf{k}}^{\alpha}\right)}\right) \delta\left(\sigma \omega_{\mathbf{k}}^{\alpha}-\sigma^{\prime} \omega_{\mathbf{k}^{\prime}}^{\beta}-\sigma^{\prime \prime} \omega_{\mathbf{k}-\mathbf{k}^{\prime}}^{\gamma}\right) \\
& -\frac{8 \pi}{\epsilon_{\|}^{\prime}\left(\mathbf{k}, \sigma \omega_{\mathbf{k}}^{\alpha}\right)} \sum_{\sigma^{\prime}, \sigma^{\prime \prime}} \sum_{\beta} \int d \mathbf{k}^{\prime} \frac{k_{i} k_{j}^{\prime}}{k k^{\prime}} \chi_{i j k}^{(2)}\left(\mathbf{k}^{\prime}, \sigma^{\prime} \omega_{\mathbf{k}^{\prime}}^{\beta} \mid \mathbf{k}-\mathbf{k}^{\prime}, \sigma^{\prime \prime} \omega_{\mathbf{k}-\mathbf{k}^{\prime}}^{T}\right) \frac{k_{n} k_{l}^{\prime}}{k k^{\prime}}\left(\delta_{k m}-\frac{\left(\mathbf{k}-\mathbf{k}^{\prime}\right)_{k}\left(\mathbf{k}-\mathbf{k}^{\prime}\right)_{m}}{\left(\mathbf{k}-\mathbf{k}^{\prime}\right)^{2}}\right) \\
& \times \chi_{n l m}^{(2) *}\left(\mathbf{k}^{\prime}, \sigma^{\prime} \omega_{\mathbf{k}^{\prime}}^{\beta} \mid \mathbf{k}-\mathbf{k}^{\prime}, \sigma^{\prime \prime} \omega_{\mathbf{k}-\mathbf{k}^{\prime}}^{T}\right)\left(\frac{1}{2} \frac{I_{\mathbf{k}-\mathbf{k}^{\prime}}^{\sigma^{\prime \prime}} I_{\mathbf{k}}^{\sigma \alpha}}{\epsilon_{\|}^{\prime}\left(\mathbf{k}^{\prime}, \sigma^{\prime} \omega_{\mathbf{k}^{\prime}}^{\beta}\right)}+\frac{I_{\mathbf{k}^{\prime}}^{\sigma^{\prime} \beta} I_{\mathbf{k}}^{\sigma \alpha}}{\Lambda_{\perp}^{\prime}\left(\mathbf{k}-\mathbf{k}^{\prime}, \sigma^{\prime \prime} \omega_{\mathbf{k}-\mathbf{k}^{\prime}}^{T}\right)}-\frac{1}{2} \frac{I_{\mathbf{k}-\mathbf{k}^{\prime}}^{\sigma^{\prime \prime} T} \epsilon_{\mathbf{k}^{\prime}}^{\sigma^{\prime} \beta}}{\epsilon_{\|}^{\prime}\left(\mathbf{k}, \sigma \omega_{\mathbf{k}}^{\alpha}\right)}\right) \delta\left(\sigma \omega_{\mathbf{k}}^{\alpha}-\sigma^{\prime} \omega_{\mathbf{k}^{\prime}}^{\beta}-\sigma^{\prime \prime} \omega_{\mathbf{k}-\mathbf{k}^{\prime}}^{T}\right) \\
& -\frac{4 \pi}{\epsilon_{\|}^{\prime}\left(\mathbf{k}, \sigma \omega_{\mathbf{k}}^{\alpha}\right)} \sum_{\sigma^{\prime}, \sigma^{\prime \prime}} \int d \mathbf{k}^{\prime} \frac{k_{i}}{k}\left(\delta_{j l}-\frac{k_{j}^{\prime} k_{l}^{\prime}}{k^{\prime 2}}\right) \chi_{i j k}^{(2)}\left(\mathbf{k}^{\prime}, \sigma^{\prime} \omega_{\mathbf{k}^{\prime}}^{T} / \mathbf{k}-\mathbf{k}^{\prime}, \sigma^{\prime \prime} \omega_{\mathbf{k}-\mathbf{k}^{\prime}}^{T}\right) \frac{k_{n}}{k}\left(\delta_{k m}-\frac{\left(\mathbf{k}-\mathbf{k}^{\prime}\right)_{k}\left(\mathbf{k}-\mathbf{k}^{\prime}\right)_{m}}{\left(\mathbf{k}-\mathbf{k}^{\prime}\right)^{2}}\right) \\
& \times \chi_{n l m}^{(2) *}\left(\mathbf{k}^{\prime}, \sigma^{\prime} \omega_{\mathbf{k}^{\prime}}^{T} / \mathbf{k}-\mathbf{k}^{\prime}, \sigma^{\prime \prime} \omega_{\mathbf{k}-\mathbf{k}^{\prime}}^{T}\right)\left(\frac{1}{2} \frac{I_{\mathbf{k}-\mathbf{k}^{\prime}}^{\sigma^{\prime \prime} T}}{\Lambda_{\perp}^{\prime}\left(\mathbf{k}^{\prime}, \sigma^{\prime} \omega_{\mathbf{k}^{\prime}}^{T}\right)}+\frac{1}{2} \frac{I_{\mathbf{k}^{\prime}}^{\sigma^{\prime} T} I_{\mathbf{k}}^{\sigma \alpha}}{\Lambda_{\perp}^{\prime}\left(\mathbf{k}-\mathbf{k}^{\prime}, \sigma^{\prime \prime} \omega_{\mathbf{k}-\mathbf{k}^{\prime}}^{T}\right)}-\frac{1}{4} \frac{I_{\mathbf{k}^{\prime}}^{\sigma^{\prime} T} I_{\mathbf{k}-\mathbf{k}^{\prime}}^{\sigma^{\prime \prime}}\left(\mathbf{k}, \sigma \omega_{\mathbf{k}}^{\alpha}\right)}{\alpha}\right) \delta\left(\sigma \omega_{\mathbf{k}}^{\alpha}-\sigma^{\prime} \omega_{\mathbf{k}^{\prime}}^{T}-\sigma^{\prime \prime} \omega_{\mathbf{k}-\mathbf{k}^{\prime}}^{T}\right) \\
& -\frac{4}{\epsilon_{\|}^{\prime}\left(\mathbf{k}, \sigma \omega_{\mathbf{k}}^{\alpha}\right)} \sum_{\sigma^{\prime}} \sum_{\beta} \operatorname{Im} \int d \mathbf{k}^{\prime}\left[\left(\frac{k_{i} k_{j}^{\prime}\left(\mathbf{k}-\mathbf{k}^{\prime}\right)_{k}}{k k^{\prime}\left|\mathbf{k}-\mathbf{k}^{\prime}\right|} \chi_{i j k}^{(2)}\left(\mathbf{k}^{\prime}, \sigma^{\prime} \omega_{\mathbf{k}^{\prime}}^{\beta} \mid \mathbf{k}-\mathbf{k}^{\prime}, \sigma \omega_{\mathbf{k}}^{\alpha}-\sigma^{\prime} \omega_{\mathbf{k}^{\prime}}^{\beta}\right)\right)^{2} \mathcal{P} \frac{2}{\epsilon_{\|}\left(\mathbf{k}-\mathbf{k}^{\prime}, \sigma \omega_{\mathbf{k}}^{\alpha}-\sigma^{\prime} \omega_{\mathbf{k}^{\prime}}^{\beta}\right)}\right. \\
& \left.+\frac{k_{i} k_{l}}{k^{2}} \frac{k_{j}^{\prime} k_{k}^{\prime}}{k^{\prime 2}} \chi_{i j k l}^{(3)}\left(\mathbf{k}^{\prime}, \sigma^{\prime} \omega_{\mathbf{k}^{\prime}}^{\beta}\left|-\mathbf{k}^{\prime},-\sigma^{\prime} \omega_{\mathbf{k}^{\prime}}^{\beta}\right| \mathbf{k}, \sigma \omega_{\mathbf{k}}^{\alpha}\right)\right] I_{\mathbf{k}^{\prime}}^{\sigma^{\prime} \beta} I_{\mathbf{k}}^{\sigma \alpha}+\frac{4}{\epsilon_{\|}^{\prime}\left(\mathbf{k}, \sigma \omega_{\mathbf{k}}^{\alpha}\right)} \sum_{\sigma^{\prime}} \sum_{\beta} \int d \mathbf{k}^{\prime} \sum_{a} \int d \mathbf{v} \\
& \times \frac{4 e_{a}^{2}}{\left|\mathbf{k}-\mathbf{k}^{\prime}\right|^{2}\left|\epsilon_{\|}\left(\mathbf{k}-\mathbf{k}^{\prime}, \sigma \omega_{\mathbf{k}}^{\alpha}-\sigma^{\prime} \omega_{\mathbf{k}^{\prime}}^{\beta}\right)\right|^{2}}\left|\frac{k_{i} k_{j}^{\prime}\left(\mathbf{k}-\mathbf{k}^{\prime}\right)_{k}}{k k^{\prime}\left|\mathbf{k}-\mathbf{k}^{\prime}\right|} \chi_{i j k}^{(2)}\left(\mathbf{k}^{\prime}, \sigma^{\prime} \omega_{\mathbf{k}^{\prime}}^{\beta} \mid \mathbf{k}-\mathbf{k}^{\prime}, \sigma \omega_{\mathbf{k}}^{\alpha}-\sigma^{\prime} \omega_{\mathbf{k}^{\prime}}^{\beta}\right)\right|^{2} \\
& \times\left(\frac{I_{\mathbf{k}^{\prime}}^{\sigma^{\prime} \beta}}{\epsilon_{\|}^{\prime}\left(\mathbf{k}, \sigma \omega_{\mathbf{k}}^{\alpha}\right)}-\frac{I_{\mathbf{k}}^{\sigma \alpha}}{\epsilon_{\|}^{\prime}\left(\mathbf{k}^{\prime}, \sigma^{\prime} \omega_{\mathbf{k}^{\prime}}^{\beta}\right)}\right) \delta\left[\sigma \omega_{\mathbf{k}}^{\alpha}-\sigma^{\prime} \omega_{\mathbf{k}^{\prime}}^{\beta}-\left(\mathbf{k}-\mathbf{k}^{\prime}\right) \cdot \mathbf{v}\right] f_{a}(\mathbf{v})
\end{aligned}
$$




$$
\begin{aligned}
& -\frac{4}{\epsilon_{\|}^{\prime}\left(\mathbf{k}, \sigma \omega_{\mathbf{k}}^{\alpha}\right)} \sum_{\sigma^{\prime}} \operatorname{Im} \int d \mathbf{k}^{\prime}\left[\left(\delta_{j m}-\frac{k_{j}^{\prime} k_{m}^{\prime}}{k^{\prime 2}}\right) \frac{k_{i}\left(\mathbf{k}-\mathbf{k}^{\prime}\right)_{k}}{k\left|\mathbf{k}-\mathbf{k}^{\prime}\right|} \chi_{i j k}^{(2)}\left(\mathbf{k}^{\prime}, \sigma^{\prime} \omega_{\mathbf{k}^{\prime}}^{T} \mid \mathbf{k}-\mathbf{k}^{\prime}, \sigma \omega_{\mathbf{k}}^{\alpha}-\sigma^{\prime} \omega_{\mathbf{k}^{\prime}}^{T}\right) \frac{k_{n}\left(\mathbf{k}-\mathbf{k}^{\prime}\right)_{l}}{k\left|\mathbf{k}-\mathbf{k}^{\prime}\right|}\right. \\
& \times \chi_{n m l}^{(2)}\left(\mathbf{k}^{\prime}, \sigma^{\prime} \omega_{\mathbf{k}^{\prime}}^{T} \mid \mathbf{k}-\mathbf{k}^{\prime}, \sigma \omega_{\mathbf{k}}^{\alpha}-\sigma^{\prime} \omega_{\mathbf{k}^{\prime}}^{T}\right) \mathcal{P} \frac{2}{\epsilon_{\|}\left(\mathbf{k}-\mathbf{k}^{\prime}, \sigma \omega_{\mathbf{k}}^{\alpha}-\sigma^{\prime} \omega_{\mathbf{k}^{\prime}}^{T}\right)}+\frac{k_{i} k_{l}}{k^{2}}\left(\delta_{j k}-\frac{k_{j}^{\prime} k_{k}^{\prime}}{k^{\prime 2}}\right) \\
& \left.+\chi_{i j k l}^{(3)}\left(\mathbf{k}^{\prime}, \sigma^{\prime} \omega_{\mathbf{k}^{\prime}}^{T}\left|-\mathbf{k}^{\prime},-\sigma^{\prime} \omega_{\mathbf{k}^{\prime}}^{T}\right| \mathbf{k}, \sigma \omega_{\mathbf{k}}^{\alpha}\right)\right] \frac{I_{\mathbf{k}^{\prime}}^{\sigma^{\prime} T}}{2} I_{\mathbf{k}}^{\sigma \alpha}+\frac{4}{\epsilon_{\|}^{\prime}\left(\mathbf{k}, \sigma \omega_{\mathbf{k}}^{\alpha}\right)} \sum_{\sigma^{\prime}} \int d \mathbf{k}^{\prime} \sum_{a} \int d \mathbf{v} \\
& \times \frac{4 e_{a}^{2}}{\left(\mathbf{k}-\mathbf{k}^{\prime}\right)^{2}\left|\epsilon_{\|}\left(\mathbf{k}-\mathbf{k}^{\prime}, \sigma \omega_{\mathbf{k}}^{\alpha}-\sigma^{\prime} \omega_{\mathbf{k}^{\prime}}^{T}\right)\right|} \frac{k_{i}\left(\mathbf{k}-\mathbf{k}^{\prime}\right)_{k}}{k\left|\mathbf{k}-\mathbf{k}^{\prime}\right|}\left(\delta_{n j}-\frac{k_{n}^{\prime} k_{j}^{\prime}}{k^{\prime 2}}\right) \chi_{i j k}^{(2)}\left(\mathbf{k}^{\prime}, \sigma^{\prime} \omega_{\mathbf{k}^{\prime}}^{T} \mid \mathbf{k}-\mathbf{k}^{\prime}, \sigma \omega_{\mathbf{k}}^{\alpha}-\sigma^{\prime} \omega_{\mathbf{k}^{\prime}}^{T}\right) \\
& \times \frac{k_{m}\left(\mathbf{k}-\mathbf{k}^{\prime}\right)_{p}}{k\left|\mathbf{k}-\mathbf{k}^{\prime}\right|} \chi_{m n p}^{(2) *}\left(\mathbf{k}^{\prime}, \sigma^{\prime} \omega_{\mathbf{k}^{\prime}}^{T} \mid \mathbf{k}-\mathbf{k}^{\prime}, \sigma \omega_{\mathbf{k}}^{\alpha}-\sigma^{\prime} \omega_{\mathbf{k}^{\prime}}^{T}\right)\left(\frac{1}{2} \frac{I_{\mathbf{k}^{\prime}}^{\sigma^{\prime} T}}{\epsilon_{\|}^{\prime}\left(\mathbf{k}, \sigma \omega_{\mathbf{k}}^{\alpha}\right)}-\frac{I_{\mathbf{k}}^{\sigma \alpha}}{\Lambda_{\perp}^{\prime}\left(\mathbf{k}^{\prime}, \sigma^{\prime} \omega_{\mathbf{k}^{\prime}}^{T}\right)}\right) \delta\left[\sigma \omega_{\mathbf{k}}^{\alpha}-\sigma^{\prime} \omega_{\mathbf{k}^{\prime}}^{T}-\left(\mathbf{k}-\mathbf{k}^{\prime}\right) \cdot \mathbf{v}\right] f_{a}(\mathbf{v}) .
\end{aligned}
$$

In the above the primed quantities associated with linear dielectric constants denote derivatives with respect to the frequency, $\epsilon_{\|}^{\prime}(\mathbf{k}, \omega)=\partial \operatorname{Re} \epsilon_{\|}(\mathbf{k}, \omega) / \partial \omega$ and $\Lambda_{\perp}^{\prime}(\mathbf{k}, \omega)=\partial \operatorname{Re}$ $\Lambda_{\perp}(\mathbf{k}, \omega) / \partial \omega$. Note that Eq. (4) is formally exact. As one can appreciate, the expression is rather complicated and is still formal in that the various nonlinear susceptibilities are yet to be specified in detail. In order to reduce the above formal result to a form that lends itself to a numerical or theoretical analysis, one must provide specific expressions for the various dielectric susceptibility tensors. When we do so, it will turn out that the first two terms on the right-hand side correspond to the induced and spontaneous emissions of the longitudinal mode $\alpha$, respectively, the next group of terms within the $\mathbf{k}^{\prime}$ integral correspond to the three-wave decay process involving three longitudinal modes $(\alpha, \beta, \gamma)$; the fol- lowing group of terms within the next $\mathbf{k}^{\prime}$ integral describe three-wave decay involving two longitudinal modes, $\alpha$ and $\beta$ and a transverse mode $T$; the next terms describe the decay process involving $(\alpha, T, T)$; the next two $\mathbf{k}^{\prime}$ integrals represent the induced and spontaneous scattering terms involving particles and two longitudinal modes, $\alpha$ and $\alpha^{\prime}$; and finally, the last two $\mathbf{k}^{\prime}$ integrals represent the induced and spontaneous scattering terms involving a longitudinal mode $\alpha$ and a transverse mode $T$.

One can find the formal wave kinetic equation for the transverse electromagnetic mode in Eq. (32) of Ref. 22. However, in writing down Eq. (32), Ref. 22 ignored certain terms in the previous Eq. (25) of the same reference. Here, we reinstate those terms that were ignored in Ref. 22 ,

$$
\begin{aligned}
& \frac{\partial I_{\mathbf{k}}^{\sigma T}}{\partial t}=-\frac{2 \operatorname{Im} \Lambda_{\perp}\left(\mathbf{k}, \sigma \omega_{\mathbf{k}}^{T}\right)}{\Lambda_{\perp}^{\prime}\left(\mathbf{k}, \sigma \omega_{\mathbf{k}}^{T}\right)} I_{\mathbf{k}}^{\sigma T}+\sum_{a} \frac{4 e_{a}^{2}}{\left|\omega_{\mathbf{k}}^{T} \Lambda_{\perp}^{\prime}\left(\mathbf{k}, \sigma \omega_{\mathbf{k}}^{T}\right)\right|^{2}} \int d \mathbf{v} \frac{(\mathbf{k} \times \mathbf{v})^{2}}{k^{2}} \delta\left(\sigma \omega_{\mathbf{k}}^{T}-\mathbf{k} \cdot \mathbf{v}\right) f_{a}(\mathbf{v}) \\
& -\frac{4 \pi}{\Lambda_{\perp}^{\prime}\left(\mathbf{k}, \sigma \omega_{\mathbf{k}}^{T}\right)} \sum_{\sigma^{\prime}, \sigma^{\prime \prime}} \sum_{\beta, \gamma} \int d \mathbf{k}^{\prime} \frac{k_{j}^{\prime}\left(\mathbf{k}-\mathbf{k}^{\prime}\right)_{k}}{k^{\prime}\left|\mathbf{k}-\mathbf{k}^{\prime}\right|}\left(\delta_{i n}-\frac{k_{i} k_{n}}{k^{2}}\right) \chi_{i j k}^{(2)}\left(\mathbf{k}^{\prime}, \sigma^{\prime} \omega_{\mathbf{k}^{\prime}}^{\beta} \mid \mathbf{k}-\mathbf{k}^{\prime}, \sigma^{\prime \prime} \omega_{\mathbf{k}-\mathbf{k}^{\prime}}^{\gamma}\right) \\
& \times \frac{k_{l}^{\prime}\left(\mathbf{k}-\mathbf{k}^{\prime}\right)_{m}}{k^{\prime}\left|\mathbf{k}-\mathbf{k}^{\prime}\right|} \chi_{n l m}^{(2) *}\left(\mathbf{k}^{\prime}, \sigma^{\prime} \omega_{\mathbf{k}^{\prime}}^{\beta} \mid \mathbf{k}-\mathbf{k}^{\prime}, \sigma^{\prime \prime} \omega_{\mathbf{k}-\mathbf{k}^{\prime}}^{\gamma}\right) \\
& \times\left(\frac{1}{2} \frac{I_{\mathbf{k}-\mathbf{k}^{\prime}}^{\sigma^{\prime \prime} \gamma} I_{\mathbf{k}}^{\sigma T}}{\epsilon_{\|}^{\prime}\left(\mathbf{k}^{\prime}, \sigma^{\prime} \omega_{\mathbf{k}^{\prime}}^{\beta}\right)}+\frac{1}{2} \frac{I_{\mathbf{k}^{\prime}}^{\sigma^{\prime} \beta} I_{\mathbf{k}}^{\sigma T}}{\epsilon_{\|}^{\prime}\left(\mathbf{k}-\mathbf{k}^{\prime}, \sigma^{\prime \prime} \omega_{\mathbf{k}-\mathbf{k}^{\prime}}^{\gamma}\right)}-\frac{I_{\mathbf{k}^{\prime}}^{\sigma^{\prime} \beta} I_{\mathbf{k}-\mathbf{k}^{\prime}}^{\sigma^{\prime \prime} \gamma}}{\Lambda_{\perp}^{\prime}\left(\mathbf{k}, \sigma \omega_{\mathbf{k}}^{T}\right)}\right) \delta\left(\sigma \omega_{\mathbf{k}}^{T}-\sigma^{\prime} \omega_{\mathbf{k}^{\prime}}^{\beta}-\sigma^{\prime \prime} \omega_{\mathbf{k}-\mathbf{k}^{\prime}}^{\gamma}\right) \\
& -\frac{8 \pi}{\Lambda_{\perp}^{\prime}\left(\mathbf{k}, \sigma \omega_{\mathbf{k}}^{T}\right)} \sum_{\sigma^{\prime}, \sigma^{\prime \prime}} \sum_{\beta} \int d \mathbf{k}^{\prime}\left(\delta_{j l}-\frac{k_{j}^{\prime} k_{l}^{\prime}}{k^{\prime 2}}\right)\left(\delta_{i n}-\frac{k_{i} k_{n}}{k^{2}}\right) \frac{\left(\mathbf{k}-\mathbf{k}^{\prime}\right)_{k}\left(\mathbf{k}-\mathbf{k}^{\prime}\right)_{m}}{\left|\mathbf{k}-\mathbf{k}^{\prime}\right|^{2}} \\
& \times \chi_{i j k}^{(2)}\left(\mathbf{k}^{\prime}, \sigma^{\prime} \omega_{\mathbf{k}^{\prime}}^{T} \mid \mathbf{k}-\mathbf{k}^{\prime}, \sigma^{\prime \prime} \omega_{\mathbf{k}-\mathbf{k}^{\prime}}^{\beta}\right) \chi_{n l m}^{*}\left(\mathbf{k}^{\prime}, \sigma^{\prime} \omega_{\mathbf{k}^{\prime}}^{T} \mid \mathbf{k}-\mathbf{k}^{\prime}, \sigma^{\prime \prime} \omega_{\mathbf{k}-\mathbf{k}^{\prime}}^{\beta}\right) \\
& \times\left(\frac{I_{\mathbf{k}^{\prime}}^{\sigma^{\prime} T} I_{\mathbf{k}-\mathbf{k}^{\prime}}^{\sigma^{\prime \prime} \beta}}{\Lambda_{\perp}^{\prime}\left(\mathbf{k}, \sigma \omega_{\mathbf{k}}^{T}\right)}-\frac{I_{\mathbf{k}-\mathbf{k}^{\prime}}^{\sigma^{\prime \prime} \beta} I_{\mathbf{k}}^{\sigma T}}{\Lambda_{\perp}^{\prime}\left(\mathbf{k}^{\prime}, \sigma^{\prime} \omega_{\mathbf{k}^{\prime}}^{T}\right)}-\frac{1}{2} \frac{I_{\mathbf{k}^{\prime}}^{\sigma^{\prime} T} I_{\mathbf{k}}^{\sigma T}}{\epsilon_{\|}^{\prime}\left(\mathbf{k}-\mathbf{k}^{\prime}, \sigma^{\prime \prime} \omega_{\mathbf{k}-\mathbf{k}^{\prime}}^{L}\right)}\right) \delta\left(\sigma \omega_{\mathbf{k}}^{T}-\sigma^{\prime} \omega_{\mathbf{k}^{\prime}}^{T}-\sigma^{\prime \prime} \omega_{\mathbf{k}-\mathbf{k}^{\prime}}^{\beta}\right) \\
& -\frac{4}{\Lambda_{\perp}^{\prime}\left(\mathbf{k}, \sigma \omega_{\mathbf{k}}^{T}\right)} \sum_{\sigma^{\prime}} \sum_{\beta} \operatorname{Im} \int d \mathbf{k}^{\prime}\left[\left(\delta_{i n}-\frac{k_{i} k_{n}}{k^{2}}\right) \frac{k_{j}^{\prime}\left(\mathbf{k}-\mathbf{k}^{\prime}\right)_{k}}{k^{\prime}\left|\mathbf{k}-\mathbf{k}^{\prime}\right|} \chi_{i j k}^{(2)}\left(\mathbf{k}^{\prime}, \sigma^{\prime} \omega_{\mathbf{k}^{\prime}}^{\beta} \mid \mathbf{k}-\mathbf{k}^{\prime}, \sigma \omega_{\mathbf{k}}^{T}-\sigma^{\prime} \omega_{\mathbf{k}^{\prime}}^{\beta}\right)\right.
\end{aligned}
$$




$$
\begin{aligned}
& \times \frac{k_{m}^{\prime}\left(\mathbf{k}-\mathbf{k}^{\prime}\right)_{l}}{k^{\prime}\left|\mathbf{k}-\mathbf{k}^{\prime}\right|} \chi_{n m l}^{(2)}\left(\mathbf{k}^{\prime}, \sigma^{\prime} \omega_{\mathbf{k}^{\prime}}^{\beta} \mid \mathbf{k}-\mathbf{k}^{\prime}, \sigma \omega_{\mathbf{k}}^{T}-\sigma^{\prime} \omega_{\mathbf{k}^{\prime}}^{\beta}\right) \mathcal{P} \frac{2}{\epsilon_{\|}\left(\mathbf{k}-\mathbf{k}^{\prime}, \sigma \omega_{\mathbf{k}}^{T}-\sigma^{\prime} \omega_{\mathbf{k}^{\prime}}^{\beta}\right)}+\left(\delta_{i l}-\frac{k_{i} k_{l}}{k^{2}}\right) \frac{k_{j}^{\prime} k_{k}^{\prime}}{k^{\prime 2}} \\
& \left.\times \chi_{i j k l}^{(3)}\left(\mathbf{k}^{\prime}, \sigma^{\prime} \omega_{\mathbf{k}^{\prime}}^{\beta}\left|-\mathbf{k}^{\prime},-\sigma^{\prime} \omega_{\mathbf{k}^{\prime}}^{\beta}\right| \mathbf{k}, \sigma \omega_{\mathbf{k}}^{T}\right)\right] I_{\mathbf{k}^{\prime}}^{\sigma^{\prime} \beta} \frac{I_{\mathbf{k}}^{\sigma T}}{2}+\frac{4}{\Lambda_{\perp}^{\prime}\left(\mathbf{k}, \sigma \omega_{\mathbf{k}}^{T}\right)} \sum_{\sigma^{\prime}} \sum_{\beta} \int d \mathbf{k}^{\prime} \sum_{a} \int d \mathbf{v} \\
& \times \frac{4 e_{a}^{2}}{\left(\mathbf{k}-\mathbf{k}^{\prime}\right)^{2}\left|\epsilon_{\|}\left(\mathbf{k}-\mathbf{k}^{\prime}, \sigma \omega_{\mathbf{k}}^{T}-\sigma^{\prime} \omega_{\mathbf{k}^{\prime}}^{\beta}\right)\right|^{2}} \frac{k_{j}^{\prime}\left(\mathbf{k}-\mathbf{k}^{\prime}\right)_{k}}{k^{\prime}\left|\mathbf{k}-\mathbf{k}^{\prime}\right|}\left(\delta_{i m}-\frac{k_{i} k_{m}}{k^{2}}\right) \chi_{i j k}^{(2)}\left(\mathbf{k}^{\prime}, \sigma^{\prime} \omega_{\mathbf{k}^{\prime}}^{\beta} \mid \mathbf{k}-\mathbf{k}^{\prime}, \sigma \omega_{\mathbf{k}}^{T}-\sigma^{\prime} \omega_{\mathbf{k}^{\prime}}^{\beta}\right) \\
& \times \frac{k_{n}^{\prime}\left(\mathbf{k}-\mathbf{k}^{\prime}\right)_{p}}{k^{\prime}\left|\mathbf{k}-\mathbf{k}^{\prime}\right|} \chi_{m n p}^{(2) *}\left(\mathbf{k}^{\prime}, \sigma^{\prime} \omega_{\mathbf{k}^{\prime}}^{\beta} \mid \mathbf{k}-\mathbf{k}^{\prime}, \sigma \omega_{\mathbf{k}}^{T}-\sigma^{\prime} \omega_{\mathbf{k}^{\prime}}^{\beta}\right)\left(\frac{I_{\mathbf{k}^{\prime}}^{\sigma^{\prime} \beta}}{\Lambda_{\perp}^{\prime}\left(\mathbf{k}, \sigma \omega_{\mathbf{k}}^{T}\right)}-\frac{1}{2} \frac{I_{\mathbf{k}}^{\sigma T}}{\epsilon_{\|}^{\prime}\left(\mathbf{k}^{\prime}, \sigma^{\prime} \omega_{\mathbf{k}^{\prime}}^{\beta}\right)}\right) \\
& \times \delta\left[\sigma \omega_{\mathbf{k}}^{T}-\sigma^{\prime} \omega_{\mathbf{k}^{\prime}}^{\beta}-\left(\mathbf{k}-\mathbf{k}^{\prime}\right) \cdot \mathbf{v}\right] f_{a}(\mathbf{v}) .
\end{aligned}
$$

Note that in Ref. 22 the first two terms on the right-hand side of Eq. (5), which represent the induced and spontaneous emissions of the fast transverse waves, were ignored at the outset. These terms can indeed be omitted since the linear wave-particle resonance condition, $\sigma \omega_{\mathbf{k}}^{T}=\mathbf{k} \cdot \mathbf{v}$ cannot be satisfied. However, we retained these terms here for the sake of completeness. In Ref. 22, the only three-wave decay terms were the first group of $\mathbf{k}^{\prime}$ integrals on the right-hand side of Eq. (5) dictated by the nonlinear wave-wave resonance condition $\delta\left(\sigma \omega_{\mathbf{k}}^{T}-\sigma^{\prime} \omega_{\mathbf{k}^{\prime}}^{\beta}-\sigma^{\prime \prime} \omega_{\mathbf{k}-\mathbf{k}^{\prime}}^{\gamma}\right)$. These terms represent the three-wave interaction among two longitudinal waves and a transverse wave. However, the second group of $\mathbf{k}^{\prime}$ integrals dictated by the resonance condition $\delta\left(\sigma \omega_{\mathbf{k}}^{T}-\sigma^{\prime} \omega_{\mathbf{k}^{\prime}}^{T}-\right.$ $\sigma^{\prime \prime}\left(\omega_{\mathbf{k}-\mathbf{k}^{\prime}}^{\beta}\right)$ was ignored at the outset. However, these terms together represent the incoherent Raman scattering process and are responsible for the third and higher harmonic radiation emissions. This is one of the reasons for the present paper, i.e., to include such a term in the general electromagnetic weak turbulence formalism. The remaining terms represent the induced and spontaneous scattering terms, i.e., nonlinear wave-particle interaction processes. The sign error associated with Ref. 22 occurred in the last group of $\mathbf{k}^{\prime}$ integrals, namely, the spontaneous scattering term. Instead of the minus sign in front of the second term within the large parenthesis in the second to the last line in Eq. (5), Ref. 22 had the plus sign.

In Eqs. (4) and (5), the various susceptibilities are defined. For the sake of completeness, we present here the formal definitions,

$$
\begin{aligned}
& \Lambda_{i j}(\mathbf{k}, \omega)=\frac{k_{i} k_{j}}{k^{2}} \epsilon_{\|}(\mathbf{k}, \omega)+\left(\delta_{i j}-\frac{k_{i} k_{j}}{k^{2}}\right) \Lambda_{\perp}(\mathbf{k}, \omega), \\
& \epsilon_{\|}(\mathbf{k}, \omega)=1+\sum_{a} \frac{4 \pi e_{a}^{2}}{m_{a} k^{2}} \int d \mathbf{v} \frac{\mathbf{k} \cdot \partial f_{a} / \partial \mathbf{v}}{\omega-\mathbf{k} \cdot \mathbf{v}+i 0}, \\
& \Lambda_{\perp}(\mathbf{k}, \omega)=\epsilon_{\perp}(\mathbf{k}, \omega)-\frac{c^{2} k^{2}}{\omega^{2}} \\
& \epsilon_{\perp}(\mathbf{k}, \omega)=1+\sum_{a} \frac{4 \pi e_{a}^{2}}{2 m_{a} \omega} \int d \mathbf{v} \frac{1}{\omega-\mathbf{k} \cdot \mathbf{v}+i 0} \frac{(\mathbf{k} \times \mathbf{v}) \times \mathbf{k}}{k^{2}} \cdot\left[\frac{\partial f_{a}}{\partial \mathbf{v}}+\frac{\mathbf{k}}{\omega} \times\left(\mathbf{v} \times \frac{\partial f_{a}}{\partial \mathbf{v}}\right)\right], \\
& \chi_{i j k}^{(2)}\left(\mathbf{k}_{1}, \omega_{1} \mid \mathbf{k}_{2}, \omega_{2}\right)=-\frac{i}{2} \sum_{a} \frac{e_{a}}{m_{a}} \frac{4 \pi e_{a}^{2}}{m_{a}\left(\omega_{1}+\omega_{2}\right)} \int d \mathbf{v} \frac{v_{i}}{\omega_{1}+\omega_{2}-\left(\mathbf{k}_{1}+\mathbf{k}_{2}\right) \cdot \mathbf{v}+i 0} \\
& \times\left\{\left[\left(1-\frac{\mathbf{k}_{1} \cdot \mathbf{v}}{\omega_{1}}\right) \frac{\partial}{\partial v_{j}}+\frac{v_{j}}{\omega_{1}} \mathbf{k}_{1} \cdot \frac{\partial}{\partial \mathbf{v}}\right] \frac{1}{\omega_{2}-\mathbf{k}_{2} \cdot \mathbf{v}+i 0}\left[\left(1-\frac{\mathbf{k}_{2} \cdot \mathbf{v}}{\omega_{2}}\right) \frac{\partial}{\partial v_{k}}+\frac{v_{k}}{\omega_{2}} \mathbf{k}_{2} \cdot \frac{\partial}{\partial \mathbf{v}}\right]+(1 \leftrightarrow 2, j \leftrightarrow k)\right\}, \\
& \chi_{i j k l}^{(3)}\left(\mathbf{k}_{1}, \omega_{1}\left|\mathbf{k}_{2}, \omega_{2}\right| \mathbf{k}_{3}, \omega_{3}\right)=\frac{(-i)^{2}}{2} \sum_{a} \frac{e_{a}^{2}}{m_{a}^{2}} \frac{4 \pi e_{a}^{2}}{m_{a} \omega} \int d \mathbf{v} \frac{v_{i}}{\omega-\mathbf{k} \cdot \mathbf{v}+i 0}\left[\left(1-\frac{\mathbf{k}_{1} \cdot \mathbf{v}}{\omega_{1}}\right) \frac{\partial}{\partial v_{j}}+\frac{v_{j}}{\omega_{1}} \mathbf{k}_{1} \cdot \frac{\partial}{\partial \mathbf{v}}\right] \\
& \times \frac{1}{\omega^{\prime}-\mathbf{k}^{\prime} \cdot \mathbf{v}+i 0}\left\{\left[\left(1-\frac{\mathbf{k}_{2} \cdot \mathbf{v}}{\omega_{2}}\right) \frac{\partial}{\partial v_{k}}+\frac{v_{k}}{\omega_{2}} \mathbf{k}_{2} \cdot \frac{\partial}{\partial \mathbf{v}}\right]\right. \\
& \left.\times \frac{1}{\omega_{3}-\mathbf{k}_{3} \cdot \mathbf{v}+i 0}\left[\left(1-\frac{\mathbf{k}_{3} \cdot \mathbf{v}}{\omega_{3}}\right) \frac{\partial}{\partial v_{l}}+\frac{v_{l}}{\omega_{3}} \mathbf{k}_{3} \cdot \frac{\partial}{\partial \mathbf{v}}\right]+(2 \leftrightarrow 3, k \leftrightarrow l)\right\},
\end{aligned}
$$


where $\omega=\omega_{1}+\omega_{2}+\omega_{3}, \mathbf{k}=\mathbf{k}_{1}+\mathbf{k}_{2}+\mathbf{k}_{3}, \omega^{\prime}=\omega_{2}+\omega_{3}$, and $\mathbf{k}^{\prime}=\mathbf{k}_{2}+\mathbf{k}_{3}$. In the above, the quantities $\chi_{i j}^{a}(\mathbf{k}, \omega)$, $\chi_{i j k}^{a(2)}\left(\mathbf{k}_{1}, \omega_{1} \mid \mathbf{k}_{2}, \omega_{2}\right)$, and $\chi_{i j k l}^{a(3)}\left(\mathbf{k}_{1}, \omega_{1}\left|\mathbf{k}_{2}, \omega_{2}\right| \mathbf{k}_{3}, \omega_{3}\right)$ are linear, second- and third-order nonlinear susceptibility tensors, whose detailed computations in various limiting cases were given in Ref. 40.

Reference 22 employs approximate forms of the various nonlinear susceptibility tensors discussed in Ref. 40 in order to reduce the various coupling coefficients in Eqs. (4) and (5). The longitudinal mode wave kinetic equation given in Ref. 22 remains unchanged as the expression found thereof is correct, except for the fact that in Ref. 22 the inverse process of incoherent Raman scattering is not included. The additional term can be computed on the basis of approximate forms of nonlinear susceptibilities given in Ref. 40. In the subsection below we present the improved wave kinetic equation for $L$ mode, which shall be followed by the $S$ mode wave kinetic equation, and the extended form of $T$ mode wave kinetic equation.

\section{A. L wave kinetic equation}

We now present the updated version of the $L$ mode wave kinetic equation that includes the inverse Raman scattering effects

$$
\begin{aligned}
& \frac{\partial I_{\mathbf{k}}^{\sigma L}}{\partial t}=\frac{4 \pi e^{2}}{m_{e} k^{2}} \int d \mathbf{v} \delta\left(\sigma \omega_{\mathbf{k}}^{L}-\mathbf{k} \cdot \mathbf{v}\right)\left(\hat{n} e^{2} f_{e}(\mathbf{v})+\pi\left(\sigma \omega_{\mathbf{k}}^{L}\right) \mathbf{k} \cdot \frac{\partial f_{e}(\mathbf{v})}{\partial \mathbf{v}} I_{\mathbf{k}}^{\sigma L}\right)+\pi \sigma \omega_{\mathbf{k}}^{L} \frac{e^{2}}{2 T_{e}^{2}} \sum_{\sigma^{\prime}, \sigma^{\prime \prime}= \pm 1} \int d \mathbf{k}^{\prime} \frac{\mu_{\mathbf{k}-\mathbf{k}^{\prime}}^{S}\left(\mathbf{k} \cdot \mathbf{k}^{\prime}\right)^{2}}{k^{2} k^{\prime 2}\left|\mathbf{k}-\mathbf{k}^{\prime}\right|^{2}} \\
& \times\left(\sigma \omega_{\mathbf{k}}^{L} I_{\mathbf{k}^{\prime}}^{\sigma^{\prime} L} \frac{I_{\mathbf{k}-\mathbf{k}^{\prime}}^{\sigma^{\prime \prime}}}{\mu_{\mathbf{k}-\mathbf{k}^{\prime}}^{S}}-\sigma^{\prime} \omega_{\mathbf{k}^{\prime}}^{L} \frac{I_{\mathbf{k}-\mathbf{k}^{\prime}}^{\sigma^{\prime \prime}}}{\mu_{\mathbf{k}-\mathbf{k}^{\prime}}^{S}} I_{\mathbf{k}}^{\sigma L}-\sigma^{\prime \prime} \omega_{\mathbf{k}-\mathbf{k}^{\prime}}^{L} I_{\mathbf{k}^{\prime}}^{\sigma^{\prime} L} I_{\mathbf{k}}^{\sigma L}\right) \delta\left(\sigma \omega_{\mathbf{k}}^{L}-\sigma^{\prime} \omega_{\mathbf{k}^{\prime}}^{L}-\sigma^{\prime \prime} \omega_{\mathbf{k}-\mathbf{k}^{\prime}}^{S}\right) \\
& +\pi \sigma \omega_{\mathbf{k}}^{L} \frac{e^{2}}{8 m_{e}^{2} \omega_{p e}^{2}} \sum_{\sigma^{\prime}, \sigma^{\prime \prime}} \int d \mathbf{k}^{\prime} \frac{\left(\mathbf{k} \times \mathbf{k}^{\prime}\right)^{2}}{k^{2} k^{\prime 2}\left|\mathbf{k}-\mathbf{k}^{\prime}\right|^{2}}\left(\frac{k^{\prime 2}}{\sigma^{\prime} \omega_{\mathbf{k}^{\prime}}^{L}}+\frac{k^{2}}{\sigma \omega_{\mathbf{k}}^{L}}\right)^{2}\left(\sigma \omega_{\mathbf{k}}^{L} \frac{I_{\mathbf{k}-\mathbf{k}^{\prime}}^{\sigma^{\prime \prime} T}}{2} I_{\mathbf{k}^{\prime}}^{\sigma^{\prime} L}-\sigma^{\prime} \omega_{\mathbf{k}^{\prime}}^{L} \frac{I_{\mathbf{k}-\mathbf{k}^{\prime}}^{\sigma^{\prime \prime} T}}{2} I_{\mathbf{k}}^{\sigma L}-\sigma^{\prime \prime} \omega_{\mathbf{k}-\mathbf{k}^{\prime}}^{T} I_{\mathbf{k}^{\prime}}^{\sigma^{\prime} L} I_{\mathbf{k}}^{\sigma L}\right) \\
& \times \delta\left(\sigma \omega_{\mathbf{k}}^{L}-\sigma^{\prime} \omega_{\mathbf{k}^{\prime}}^{L}-\sigma^{\prime \prime} \omega_{\mathbf{k}-\mathbf{k}^{\prime}}^{T}\right)+\pi \sigma \omega_{\mathbf{k}}^{L} \frac{e^{2}}{4 m_{e}^{2}} \sum_{\sigma^{\prime}, \sigma^{\prime \prime}} \int d \mathbf{k}^{\prime} \frac{k^{2}}{\left(\omega_{\mathbf{k}^{\prime}}^{T}\right)^{2}\left(\omega_{\mathbf{k}-\mathbf{k}^{\prime}}^{T}\right)^{2}} \\
& \times\left(1+\frac{\left[\mathbf{k}^{\prime} \cdot\left(\mathbf{k}-\mathbf{k}^{\prime}\right)\right]^{2}}{k^{2}\left|\mathbf{k}-\mathbf{k}^{\prime}\right|^{2}}\right)\left(\sigma \omega_{\mathbf{k}}^{L} \frac{I_{\mathbf{k}^{\prime}}^{\sigma^{\prime} T}}{2} \frac{I_{\mathbf{k}-\mathbf{k}^{\prime}}^{\sigma^{\prime \prime} T}}{2}-\sigma^{\prime} \omega_{\mathbf{k}^{\prime}}^{T} \frac{I_{\mathbf{k}-\mathbf{k}^{\prime}}^{\sigma^{\prime \prime} T}}{2} I_{\mathbf{k}}^{\sigma L}-\sigma^{\prime \prime} \omega_{\mathbf{k}-\mathbf{k}^{\prime}}^{T} \frac{I_{\mathbf{k}^{\prime}}^{\sigma^{\prime} T}}{2} I_{\mathbf{k}}^{\sigma L}\right) \\
& \times \delta\left(\sigma \omega_{\mathbf{k}}^{L}-\sigma^{\prime} \omega_{\mathbf{k}^{\prime}}^{T}-\sigma^{\prime \prime} \omega_{\mathbf{k}-\mathbf{k}^{\prime}}^{T}\right)+\pi \sigma \omega_{\mathbf{k}}^{L} \frac{e^{2}}{2 T_{e}^{2}} \sum_{\sigma^{\prime}, \sigma^{\prime \prime}} \int d \mathbf{k}^{\prime} \frac{\mu_{\mathbf{k}^{\prime}}^{S}\left(\mathbf{k} \times \mathbf{k}^{\prime}\right)^{2}}{k^{2} k^{\prime 2}\left|\mathbf{k}-\mathbf{k}^{\prime}\right|^{2}} \\
& \times\left(\sigma \omega_{\mathbf{k}}^{L} \frac{I_{\mathbf{k}-\mathbf{k}^{\prime}}^{\sigma^{\prime \prime} T}}{2} \frac{I_{\mathbf{k}^{\prime}}^{\sigma^{\prime} S}}{\mu_{\mathbf{k}^{\prime}}^{S}}-\sigma^{\prime} \omega_{\mathbf{k}^{\prime}}^{L} \frac{I_{\mathbf{k}-\mathbf{k}^{\prime}}^{\sigma^{\prime \prime} T}}{2} I_{\mathbf{k}}^{\sigma L}-\sigma^{\prime \prime} \omega_{\mathbf{k}-\mathbf{k}^{\prime}}^{T} \frac{I_{\mathbf{k}^{\prime}}^{\sigma^{\prime} S}}{\mu_{\mathbf{k}^{\prime}}^{S}} I_{\mathbf{k}}^{\sigma L}\right) \delta\left(\sigma \omega_{\mathbf{k}}^{L}-\sigma^{\prime} \omega_{\mathbf{k}^{\prime}}^{S}-\sigma^{\prime \prime} \omega_{\mathbf{k}-\mathbf{k}^{\prime}}^{T}\right) \\
& -\sigma \omega_{\mathbf{k}}^{L} \frac{e^{2}}{\hat{n} m_{e}^{2} \omega_{p e}^{2}} \sum_{\sigma^{\prime}} \int d \mathbf{k}^{\prime} \int d \mathbf{v} \frac{\left(\mathbf{k} \cdot \mathbf{k}^{\prime}\right)^{2}}{k^{2} k^{\prime 2}} \delta\left[\sigma \omega_{\mathbf{k}}^{L}-\sigma^{\prime} \omega_{\mathbf{k}^{\prime}}^{L}-\left(\mathbf{k}-\mathbf{k}^{\prime}\right) \cdot \mathbf{v}\right] \\
& \times\left[\frac{\hat{n} e^{2}}{\omega_{p e}^{2}}\left(\sigma^{\prime} \omega_{\mathbf{k}^{\prime}}^{L} I_{\mathbf{k}}^{\sigma L}-\sigma \omega_{\mathbf{k}}^{L} I_{\mathbf{k}^{\prime}}^{\sigma^{\prime} L}\right)\left[f_{e}(\mathbf{v})+f_{i}(\mathbf{v})\right]-\pi \frac{m_{e}}{m_{i}} I_{\mathbf{k}^{\prime}}^{\sigma^{\prime} L} I_{\mathbf{k}}^{\sigma L}\left(\mathbf{k}-\mathbf{k}^{\prime}\right) \cdot \frac{\partial f_{i}(\mathbf{v})}{\partial \mathbf{v}}\right] \\
& -\sigma \omega_{\mathbf{k}}^{L} \frac{e^{2}}{\hat{n} m_{e}^{2} \omega_{p e}^{2}} \sum_{\sigma^{\prime}} \int d \mathbf{k}^{\prime} \int d \mathbf{v} \frac{\left(\mathbf{k} \times \mathbf{k}^{\prime}\right)^{2}}{k^{2} k^{\prime 2}} \delta\left[\sigma \omega_{\mathbf{k}}^{L}-\sigma^{\prime} \omega_{\mathbf{k}^{\prime}}^{T}-\left(\mathbf{k}-\mathbf{k}^{\prime}\right) \cdot \mathbf{v}\right] \\
& \times\left[\frac{\hat{n} e^{2}}{\omega_{p e}^{2}}\left(\sigma^{\prime} \omega_{\mathbf{k}^{\prime}}^{T} I_{\mathbf{k}}^{\sigma L}-\sigma \omega_{\mathbf{k}}^{L} \frac{I_{\mathbf{k}^{\prime}}^{\sigma^{\prime} T}}{2}\right)\left[f_{e}(\mathbf{v})+f_{i}(\mathbf{v})\right]-\pi \frac{m_{e}}{m_{i}} \frac{I_{\mathbf{k}^{\prime}}^{\sigma^{\prime} T}}{2} I_{\mathbf{k}}^{\sigma L}\left(\mathbf{k}-\mathbf{k}^{\prime}\right) \cdot \frac{\partial f_{i}(\mathbf{v})}{\partial \mathbf{v}}\right],
\end{aligned}
$$

where

$$
\mu_{\mathbf{k}}^{S}=|k|^{3} \lambda_{D e}^{3}\left(\frac{m_{e}}{m_{i}}\right)^{1 / 2}\left(1+\frac{3 T_{i}}{T_{e}}\right)^{1 / 2}
$$

Note that the first velocity integral term on the right-hand side of Eq. (7) that contains the resonance factor $\delta\left(\sigma \omega_{\mathbf{k}}^{L}-\mathbf{k} \cdot \mathbf{v}\right)$ (i.e., linear wave-particle interaction) represents the spontane- ous and induced emissions of $L$ waves; the second $\mathbf{k}^{\prime}$ integral term dictated by the three-wave resonance condition $\delta\left(\sigma \omega_{\mathbf{k}}^{L}-\sigma^{\prime} \omega_{\mathbf{k}^{\prime}}^{L}-\sigma^{\prime \prime} \omega_{\mathbf{k}-\mathbf{k}^{\prime}}^{S}\right)$ (nonlinear three-wave interaction) represents the decay/coalescence involving $L$ mode with another $L$ mode and an $S$ mode; the next $\mathbf{k}^{\prime}$-integral associated with $\delta\left(\sigma \omega_{\mathbf{k}}^{L}-\sigma^{\prime} \omega_{\mathbf{k}^{\prime}}^{L}-\sigma^{\prime \prime} \omega_{\mathbf{k}-\mathbf{k}^{\prime}}^{T}\right)$ represents the decay/coalescence of two $L$ modes into a $T$ mode at twice the plasma frequency (a process related to the harmonic emission); the 
third $\mathbf{k}^{\prime}$-integral associated with $\delta\left(\sigma \omega_{\mathbf{k}}^{L}-\sigma^{\prime} \omega_{\mathbf{k}^{\prime}}^{T}-\sigma^{\prime \prime} \omega_{\mathbf{k}-\mathbf{k}^{\prime}}^{T}\right)$ represents the decay/coalescence of two $T$ modes into an $L$ mode. This is the reverse process of the incoherent Raman scattering term that was missing in Ref. 22; the fourth $\mathbf{k}^{\prime}$-integral with the factor $\delta\left(\sigma \omega_{\mathbf{k}}^{L}-\sigma^{\prime} \omega_{\mathbf{k}^{\prime}}^{S}-\sigma^{\prime \prime} \omega_{\mathbf{k}-\mathbf{k}^{\prime}}^{T}\right)$ corresponds to the decay/coalescence process involving $L, S$, and a transverse mode $T$ at the plasma frequency (this is a reverse process for one of the processes for the fundamental emission); the double integral term $\int d \mathbf{v} \int d \mathbf{k}^{\prime} \cdots$ dictated by the nonlinear wave-particle resonance condition $\delta\left[\sigma \omega_{\mathbf{k}}^{L}-\sigma^{\prime} \omega_{\mathbf{k}^{\prime}}^{L}-(\mathbf{k}-\right.$ $\left.\left.\mathbf{k}^{\prime}\right) \cdot \mathbf{v}\right]$ (nonlinear wave-particle interaction) represents the spontaneous and induced scattering processes involving two Langmuir waves and the particles, and the similar term which contains $\delta\left[\sigma \omega_{\mathbf{k}}^{L}-\sigma^{\prime} \omega_{\mathbf{k}^{\prime}}^{T}-\left(\mathbf{k}-\mathbf{k}^{\prime}\right) \cdot \mathbf{v}\right]$ corresponds to spontaneous and induced scattering processes involving $L$ and $T$ modes and the particles - this is another process that contributes to the fundamental emission.

\section{B. S wave kinetic equation}

Moving on the ion-sound mode, $\alpha=S$, the expression found in Ref. 22 remains unmodified

$$
\begin{aligned}
& \frac{\partial}{\partial t} \frac{I_{\mathbf{k}}^{\sigma S}}{\mu_{\mathbf{k}}^{S}}=\mu_{\mathbf{k}}^{S} \frac{4 \pi e^{2}}{m_{e} k^{2}} \int d \mathbf{v} \delta\left(\sigma \omega_{\mathbf{k}}^{S}-\mathbf{k} \cdot \mathbf{v}\right)\left[\hat{n} e^{2}\left[f_{e}(\mathbf{v})+f_{i}(\mathbf{v})\right]+\pi\left(\sigma \omega_{\mathbf{k}}^{L}\right)\left(\mathbf{k} \cdot \frac{\partial f_{e}(\mathbf{v})}{\partial \mathbf{v}}+\frac{m_{e}}{m_{i}} \mathbf{k} \cdot \frac{\partial f_{i}(\mathbf{v})}{\partial \mathbf{v}}\right) \frac{I_{\mathbf{k}}^{\sigma S}}{\mu_{\mathbf{k}}^{S}}\right] \\
& +\pi \sigma \omega_{\mathbf{k}}^{L} \frac{e^{2}}{4 T_{e}^{2}} \sum_{\sigma^{\prime}, \sigma^{\prime \prime}} \int d \mathbf{k}^{\prime} \frac{\mu_{\mathbf{k}}^{S}\left[\mathbf{k}^{\prime} \cdot\left(\mathbf{k}-\mathbf{k}^{\prime}\right)\right]^{2}}{k^{2} k^{\prime 2}\left|\mathbf{k}-\mathbf{k}^{\prime}\right|^{2}}\left(\sigma \omega_{\mathbf{k}}^{L} I_{\mathbf{k}^{\prime}}^{\sigma^{\prime} L} I_{\mathbf{k}-\mathbf{k}^{\prime}}^{\sigma^{\prime \prime} L}-\sigma^{\prime} \omega_{\mathbf{k}^{\prime}}^{L} I_{\mathbf{k}-\mathbf{k}^{\prime}}^{\sigma^{\prime \prime} L} \frac{I_{\mathbf{k}}^{\sigma S}}{\mu_{\mathbf{k}}^{S}}-\sigma^{\prime \prime} \omega_{\mathbf{k}-\mathbf{k}^{\prime}}^{L} I_{\mathbf{k}^{\prime}}^{\sigma^{\prime} L} \frac{I_{\mathbf{k}}^{\sigma S}}{\mu_{\mathbf{k}}^{S}}\right) \delta\left(\sigma \omega_{\mathbf{k}}^{S}-\sigma^{\prime} \omega_{\mathbf{k}^{\prime}}^{L}-\sigma^{\prime \prime} \omega_{\mathbf{k}-\mathbf{k}^{\prime}}^{L}\right) \\
& +\pi \sigma \omega_{\mathbf{k}}^{L} \frac{e^{2}}{2 T_{e}^{2}} \sum_{\sigma^{\prime}, \sigma^{\prime \prime}} \int d \mathbf{k}^{\prime} \frac{\mu_{\mathbf{k}}^{S}\left(\mathbf{k} \times \mathbf{k}^{\prime}\right)^{2}}{k^{2} k^{\prime 2}\left|\mathbf{k}-\mathbf{k}^{\prime}\right|^{2}}\left(\sigma \omega_{\mathbf{k}}^{L} \frac{I_{\mathbf{k}-\mathbf{k}^{\prime}}^{\sigma^{\prime \prime} T}}{2} I_{\mathbf{k}^{\prime}}^{\sigma^{\prime} L}-\sigma^{\prime} \omega_{\mathbf{k}^{\prime}}^{L} \frac{I_{\mathbf{k}-\mathbf{k}^{\prime}}^{\sigma^{\prime \prime} T}}{2} \frac{\sigma_{\mathbf{k}}^{\sigma S}}{\mu_{\mathbf{k}}^{S}}-\sigma^{\prime \prime} \omega_{\mathbf{k}-\mathbf{k}^{\prime}}^{T} I_{\mathbf{k}^{\prime}}^{\sigma^{\prime} L} \frac{I_{\mathbf{k}}^{\sigma S}}{\mu_{\mathbf{k}}^{S}}\right) \delta\left(\sigma \omega_{\mathbf{k}}^{S}-\sigma^{\prime} \omega_{\mathbf{k}^{\prime}}^{L}-\sigma^{\prime \prime} \omega_{\mathbf{k}-\mathbf{k}^{\prime}}^{T}\right) .
\end{aligned}
$$

The first velocity integral term on the right-hand side of Eq. (9) that contains the resonance factor $\delta\left(\sigma \omega_{\mathbf{k}}^{S}-\mathbf{k} \cdot \mathbf{v}\right)$ represents the spontaneous and induced emissions of $S$ waves; the first $\mathbf{k}^{\prime}$-integral term dictated by the three-wave resonance condition $\delta\left(\sigma \omega_{\mathbf{k}}^{S}-\sigma^{\prime} \omega_{\mathbf{k}^{\prime}}^{L}-\sigma^{\prime \prime} \omega_{\mathbf{k}-\mathbf{k}^{\prime}}^{S}\right)$ corresponds to the decay/coalescence involving $S$ mode with two $L$ modes; the next $\mathbf{k}^{\prime}$-integral associated with $\delta\left(\sigma \omega_{\mathbf{k}}^{S}-\sigma^{\prime} \omega_{\mathbf{k}^{\prime}}^{L}-\right.$ $\left.\sigma^{\prime \prime} \omega_{\mathbf{k}-\mathbf{k}^{\prime}}^{T}\right)$ represents the decay/coalescence involving an $S$ and $L$ modes and a $T$ mode (this is a third process related to the fundamental emission). Note that the right-hand side of $S$ mode wave kinetic equation given in Ref. 22 contains the spontaneous and induced scattering processes involving $S$ modes. However, since these processes are extremely slow processes we have decided to ignore such a process here.

\section{T wave kinetic equation}

For the transverse mode $T$, as noted before, the expression found in Ref. 22 contains a sign error. This occurs in association with the spontaneous scattering term, namely, the last group of $\mathbf{k}^{\prime}$ integrals in Eq. (5). We also now extend the expression found in Ref. 22 to include the incoherent Raman scattering term associated with the threewave interaction among two transverse waves and a Langmuir wave. After such a correction and the extension are taken into account, the correct $T$ mode wave kinetic equation emerges

$$
\begin{aligned}
\frac{\partial}{\partial t} \frac{I_{\mathbf{k}}^{\sigma T}}{2}= & \frac{\pi^{2} e^{2}}{2 m_{e}} \int d \mathbf{v} \frac{(\mathbf{k} \times \mathbf{v})^{2}}{k^{2}} \delta\left(\sigma \omega_{\mathbf{k}}^{T}-\mathbf{k} \cdot \mathbf{v}\right)\left(\frac{m_{e}}{4 \pi^{2}} f_{e}(\mathbf{v})+\frac{1}{\sigma \omega_{\mathbf{k}}^{T}} \mathbf{k} \cdot \frac{\partial f_{e}(\mathbf{v})}{\partial \mathbf{v}} \frac{I_{\mathbf{k}}^{\sigma T}}{2}\right)+\pi \sigma \omega_{\mathbf{k}}^{T} \frac{e^{2}}{32 m_{e}^{2} \omega_{p e}^{2}} \sum_{\sigma^{\prime}, \sigma^{\prime \prime}} \int d \mathbf{k}^{\prime} \frac{\left(\mathbf{k} \times \mathbf{k}^{\prime}\right)^{2}}{k^{2} k^{\prime 2}\left|\mathbf{k}-\mathbf{k}^{\prime}\right|^{2}} \\
& \times\left(\frac{k^{\prime 2}}{\sigma^{\prime} \omega_{\mathbf{k}^{\prime}}^{L}}-\frac{\left|\mathbf{k}-\mathbf{k}^{\prime}\right|^{2}}{\sigma^{\prime \prime} \omega_{\mathbf{k}-\mathbf{k}^{\prime}}^{L}}\right)^{2}\left(\sigma \omega_{\mathbf{k}}^{T} I_{\mathbf{k}^{\prime}}^{\sigma^{\prime} L} I_{\mathbf{k}-\mathbf{k}^{\prime}}^{\sigma^{\prime \prime} L}-\sigma^{\prime} \omega_{\mathbf{k}^{\prime}}^{L} I_{\mathbf{k}-\mathbf{k}^{\prime}}^{\sigma^{\prime \prime} L} \frac{I_{\mathbf{k}}^{\sigma T}}{2}-\sigma^{\prime \prime} \omega_{\mathbf{k}-\mathbf{k}^{\prime}}^{L} I_{\mathbf{k}^{\prime}}^{\sigma^{\prime} L} \frac{I_{\mathbf{k}}^{\sigma T}}{2}\right) \\
& \times \delta\left(\sigma \omega_{\mathbf{k}}^{T}-\sigma^{\prime} \omega_{\mathbf{k}^{\prime}}^{L}-\sigma^{\prime \prime} \omega_{\mathbf{k}-\mathbf{k}^{\prime}}^{L}\right)+\pi \sigma \omega_{\mathbf{k}}^{T} \frac{e^{2}}{4 T_{e}^{2}} \sum_{\sigma^{\prime}, \sigma^{\prime \prime}} \int d \mathbf{k}^{\prime} \frac{\mu_{\mathbf{k}-\mathbf{k}^{\prime}}^{S}\left(\mathbf{k} \times \mathbf{k}^{\prime}\right)^{2}}{k^{2} k^{\prime 2}\left|\mathbf{k}-\mathbf{k}^{\prime}\right|^{2}} \\
& \times\left(\sigma \omega_{\mathbf{k}}^{T} I_{\mathbf{k}^{\prime}}^{\sigma^{\prime} L} \frac{I_{\mathbf{k}-\mathbf{k}^{\prime}}^{\sigma^{\prime \prime} S}}{\mu_{\mathbf{k}-\mathbf{k}^{\prime}}^{S}}-\sigma^{\prime} \omega_{\mathbf{k}^{\prime}}^{L} \frac{I_{\mathbf{k}-\mathbf{k}^{\prime}}^{\sigma^{\prime \prime} S}}{\mu_{\mathbf{k}-\mathbf{k}^{\prime}}^{S}} \frac{I_{\mathbf{k}}^{\sigma T}}{2}-\sigma^{\prime \prime} \omega_{\mathbf{k}-\mathbf{k}^{\prime}}^{L} I_{\mathbf{k}^{\prime}}^{\sigma^{\prime} L} \frac{I_{\mathbf{k}}^{\sigma T}}{2}\right) \delta\left(\sigma \omega_{\mathbf{k}}^{T}-\sigma^{\prime} \omega_{\mathbf{k}^{\prime}}^{L}-\sigma^{\prime \prime} \omega_{\mathbf{k}-\mathbf{k}^{\prime}}^{S}\right)+\pi \sigma \omega_{\mathbf{k}}^{T} \frac{e^{2}}{4 m_{e}^{2}} \sum_{\sigma^{\prime}, \sigma^{\prime \prime}} \int d \mathbf{k}^{\prime} \frac{\left|\mathbf{k}-\mathbf{k}^{\prime}\right|^{2}}{\left(\omega_{\mathbf{k}}^{T}\right)^{2}\left(\omega_{\mathbf{k}^{\prime}}^{T}\right)^{2}} \\
& \times\left(1+\frac{\left(\mathbf{k} \cdot \mathbf{k}^{\prime}\right)^{2}}{k^{2} k^{\prime 2}}\right)\left(\sigma \omega_{\mathbf{k}}^{T} \frac{I_{\mathbf{k}^{\prime}}^{\sigma^{\prime} T}}{2} I_{\mathbf{k}-\mathbf{k}^{\prime}}^{\sigma^{\prime \prime} L}-\sigma^{\prime} \omega_{\mathbf{k}^{\prime}}^{T} I_{\mathbf{k}-\mathbf{k}^{\prime}}^{\sigma^{\prime \prime} L} \frac{I_{\mathbf{k}}^{\sigma T}}{2}-\sigma^{\prime \prime} \omega_{\mathbf{k}-\mathbf{k}^{\prime}}^{L} \frac{I_{\mathbf{k}^{\prime}}^{\sigma^{\prime} T}}{2} \frac{I_{\mathbf{k}}^{\sigma T}}{2}\right)
\end{aligned}
$$




$$
\begin{aligned}
& \times \delta\left(\sigma \omega_{\mathbf{k}}^{T}-\sigma^{\prime} \omega_{\mathbf{k}^{\prime}}^{T}-\sigma^{\prime \prime} \omega_{\mathbf{k}-\mathbf{k}^{\prime}}^{L}\right)-\sigma \omega_{\mathbf{k}}^{T} \frac{e^{2}}{2 \hat{n} m_{e}^{2} \omega_{p e}^{2}} \sum_{\sigma^{\prime}} \int d \mathbf{k}^{\prime} \int d \mathbf{v} \frac{\left(\mathbf{k} \times \mathbf{k}^{\prime}\right)^{2}}{k^{2} k^{\prime 2}} \\
& \times \delta\left[\sigma \omega_{\mathbf{k}}^{T}-\sigma^{\prime} \omega_{\mathbf{k}^{\prime}}^{L}-\left(\mathbf{k}-\mathbf{k}^{\prime}\right) \cdot \mathbf{v}\right]\left[\frac{\hat{n} e^{2}}{\omega_{p e}^{2}}\left(\sigma^{\prime} \omega_{\mathbf{k}^{\prime}}^{L} \frac{I_{\mathbf{k}}^{\sigma T}}{2}-\sigma \omega_{\mathbf{k}}^{T} I_{\mathbf{k}^{\prime}}^{\sigma^{\prime} L}\right)\left[f_{e}(\mathbf{v})+f_{i}(\mathbf{v})\right]-\pi \frac{m_{e}}{m_{i}} I_{\mathbf{k}^{\prime}}^{\sigma^{\prime} L} \frac{I_{\mathbf{k}}^{\sigma T}}{2}\left(\mathbf{k}-\mathbf{k}^{\prime}\right) \cdot \frac{\partial f_{i}(\mathbf{v})}{\partial \mathbf{v}}\right] .
\end{aligned}
$$

The first velocity integral term on the right-hand side of Eq. (10) that contains the resonance factor $\delta\left(\sigma \omega_{\mathbf{k}}^{T}-\mathbf{k} \cdot \mathbf{v}\right)$ corresponds to the spontaneous and induced emissions of $T$ waves. However, since the particles cannot have velocity higher than the speed of light in vacuo, while the transverse waves are superluminal modes, the wave-particle resonance cannot be satisfied. For this reason Ref. 22 had ignored these terms at the outset. This term is included here only for the sake of completeness. Ignoring such a term at the outset on physical ground is justifiable. The second $\mathbf{k}^{\prime}$-integral terms dictated by the three-wave resonance condition $\delta\left(\sigma \omega_{\mathbf{k}}^{T}-\right.$ $\left.\sigma^{\prime} \omega_{\mathbf{k}^{\prime}}^{L}-\sigma^{\prime \prime} \omega_{\mathbf{k}-\mathbf{k}^{\prime}}^{L}\right)$ represents the coalescence of two $L$ modes into a $T$ mode at the second harmonic plasma frequency (the harmonic emission); the next $\mathbf{k}^{\prime}$-integrals associated with the factor $\delta\left(\sigma \omega_{\mathbf{k}}^{T}-\sigma^{\prime} \omega_{\mathbf{k}^{\prime}}^{L}-\sigma^{\prime \prime} \omega_{\mathbf{k}-\mathbf{k}^{\prime}}^{S}\right)$ describe the merging of $L$ and $S$ modes into a $T$ mode at the fundamental plasma frequency (the first of the two mechanisms responsible for the fundamental emission). The third $\mathbf{k}^{\prime}$-integrals with $\delta\left(\sigma \omega_{\mathbf{k}}^{T}-\right.$ $\sigma^{\prime} \omega_{\mathbf{k}^{\prime}}^{T}-\sigma^{\prime \prime}\left(\omega_{\mathbf{k}-\mathbf{k}^{\prime}}^{L}\right)$ represents the extension of Ref. 22 in that these terms depict the merging of a $T$ mode and an $L$ mode into the next higher harmonic $T$ mode. This term is responsible for emissions at the third and higher harmonics, and it corresponds to the incoherent version of the Raman scattering. The double integral term $\int d \mathbf{v} \int d \mathbf{k}^{\prime} \cdots$ dictated by the nonlinear wave-particle resonance condition $\delta\left[\sigma \omega_{\mathbf{k}}^{T}-\sigma^{\prime} \omega_{\mathbf{k}^{\prime}}^{L}-(\mathbf{k}-\right.$ $\left.\mathbf{k}^{\prime}\right) \cdot \mathbf{v}$ ] (nonlinear wave-particle interaction) represents the spontaneous and induced scattering process involving $T$ and $L$ modes and the particles (this is the second process that contributes to the fundamental emission).

\section{CONCLUSION}

To conclude, in the present paper, we have revisited the problem of fully electromagnetic weak turbulence theory discussed in Ref. 22. The statistical mechanical reformulation that can be found in the above reference contains a set of fully self-consistent equations that may be analyzed by numerical means or by approximate analytical method. However, the wave kinetic equation for the transverse wave not only has a sign mistake associated with the spontaneous scattering term, but it also ignores the nonlinear three-wave interaction that involves two transverse waves and a Langmuir wave, which is the incoherent analogue of the Raman scattering process in a plasma. Such a process may account for the third and higher-harmonic plasma emissions. The present paper corrects the said sign mistake. It also extends the wave kinetic equation to allow the third- and higherharmonic emissions. The detailed numerical as well as approximate analytical analyses of the equations presented in the present paper shall be the subject of future research.

\section{ACKNOWLEDGMENTS}

This research was supported in part by NSF Grant No. AGS0940985 to the University of Maryland, in part by the Korean Ministry of Education, Science and Technology, under WCU Grant No. R31-10016 to Kyung Hee University, Korea, and by Brazilian agencies CNPq and FAPERGS.

${ }^{1}$ B. B. Kadomtsev, Plasma Turbulence (Academic, New York, 1965).

${ }^{2}$ A. A. Vedenov, Theory of Turbulent Plasma (Elsevier, New York, 1968).

${ }^{3}$ R. Z. Sagdeev and A. A. Galeev, Nonlinear Plasma Theory (Benjamin, New York, 1969).

${ }^{4}$ V. N. Tsytovich, Nonlinear Effects in a Plasma (Plenum, New York, 1970).

${ }^{5}$ R. C. Davidson, Methods in Nonlinear Plasma Theory (Academic, New York, 1972).

${ }^{6}$ S. A. Kaplan and V. N. Tsytovich, Plasma Astrophysics (Pergamon, Oxford, 1973).

${ }^{7}$ A. Hasegawa, Plasma Instabilities and Nonlinear Effects (Springer, New York, 1975).

${ }^{8}$ A. I. Akhiezer, I. A. Akhiezer, R. V. Polovin, A. G. Sitenko, and K. N. Stepanov, Plasma Electrodynamics. Vol. 2. Nonlinear Theory and Fluctuations (Pergamon, New York, 1975).

${ }^{9} \mathrm{~V}$. N. Tsytovich, An Introduction to the Theory of Plasma Turbulence (Pergamon, New York, 1977).

${ }^{10} \mathrm{~V}$. N. Tsytovich, Theory of Turbulent Plasma (Consultants Bureau, New York, 1977).

${ }^{11}$ D. B. Melrose, Plasma Astrophysics (Gordon and Breach, New York, 1980).

${ }^{12}$ A. G. Sitenko, Fluctuations and Nonlinear Wave Interactions in Plasmas (Pergamon, New York, 1982).

${ }^{13}$ D. R. Nicholson, Introduction to Plasma Theory (Wiley, New York, 1983).

${ }^{14}$ D. B. Melrose, Instabilities in Space and Laboratory Plasmas (Cambridge University Press, Cambridge, 1986).

${ }^{15}$ A. G. Sitenko and V. Malnev, Plasma Physics Theory (Chapman and Hall, New York, 1995).

${ }^{16} \mathrm{~V}$. N. Tsytovich, Lectures on Nonlinear Plasma Kinetics (Springer-Verlag, New York, 1995).

${ }^{17}$ B. B. Kadomtsev and V. I. Petviashvili, Sov. Phys. JETP 16, 1578 (1963).

${ }^{18}$ L. M. Gorbunov, V. V. Pustovalov, and V. P. Silin, Sov. Phys. JETP 20, 967 (1965)

${ }^{19}$ P. N. Kovrizhnykh and V. N. Tsytovich, Sov. Phys. JETP 20, 978 (1965).

${ }^{20}$ L. M. Al'tshul' and V. I. Karpman, Sov. Phys. JETP 20, 1043 (1965).

${ }^{21}$ D. B. Melrose, Aust. J. Phys. 23, 871 (1970).

${ }^{22}$ P. H. Yoon, Phys. Plasmas 13, 022302 (2006).

${ }^{23}$ V. N. Tsytovich, Sov. Phys. Usp. 9, 805 (1967).

${ }^{24}$ D. B. Melrose and W. Sy, Astrophys. Space Sci. 17, 343 (1972).

${ }^{25}$ D. B. Melrose and W. N. Sy, Aust. J. Phys. 25, 387 (1972).

${ }^{26}$ D. B. Melrose and J. E. Stenhouse, Astron. Astrophys. 73, 151 (1979).

${ }^{27}$ D. B. Melrose, Aust. J. Phys. 35, 67 (1982).

${ }^{28}$ D. B. Melrose, in Solar Radiophysics, edited by D. J. McLean and N. R. Labrum (Cambridge University Press, New York, 1985), pp.177-210.

${ }^{29}$ I. H. Cairns, J. Plasma Phys. 38, 169-208 (1987).

${ }^{30}$ I. H. Cairns, J. Geophys. Res. 93, 858, doi:10.1029/JA093iA02p00858 (1988).

${ }^{31}$ A. J. Willes, P. A. Robinson, and D. B. Melrose, Phys. Plasmas 3, 149 (1996).

${ }^{32}$ A. J. Willes and P. A. Robinson, Solar Phys. 170, 341 (1997).

${ }^{33}$ A. J. Willes and D. B. Melrose, Solar Phys. 171, 393 (1997).

${ }^{34}$ S. D. Edney and P. A. Robinson, Phys. Plasmas 6, 3799 (1999). 
${ }^{35}$ S. D. Edney and P. A. Robinson, Phys. Plasmas 8, 428 (2001).

${ }^{36}$ A. M. Vásquez, D. O. Gómez, and C. F. Fontán, Astrophys. J. 564, 1035 (2002).

${ }^{37}$ B. Li, A. J. Willes, P. A. Robinson, and I. H. Cairns, Phys. Plasmas 12, 052324 (2005).
${ }^{38}$ B. Li, R. A. Robinson, and I. H. Cairns, Phys. Rev. Lett. 96, 145005 (2006). ${ }^{39}$ B. Li, I. H. Cairns, and P. A. Robinson, J. Geophys. Res. 113, A06104, A06105, doi:10.1029/2007JA012958 (2008); B. Li, I. H. Cairns, and P. A. Robinson, ibid. 114, A02104, doi:10.1029/2008JA013687 (2009).

${ }^{40}$ P. H. Yoon, Phys. Plasmas 12, 112306 (2005). 